\title{
Serial Recordings of Multimodality Evoked Potentials in Multiple Sclerosis: A Four Year Follow-Up Study
}

\author{
Vicente J. Iragui, Wigbert C. Wiederholt, and John S. Romine
}

\begin{abstract}
Pattern reversal visual, brain-stem auditory, and short latency median nerve somatosensory evoked potentials (EPs) were evaluated in a prospective study over 4 years in 20 patients with clinically definite sclerosis (MS). Standardized neurological examinations were done at regular intervals and correlated with EP findings. The highest incidence of EP abnormalities occurred in the visual system followed by the somatosensory and auditory systems. Clinical relapse was usually accompanied by EP deterioration, but clinical improvement often occurred without parallel EP recovery. EP changes were not always related to clinical symptoms and often took place during remission periods in the absence of clinical changes. There was no significant correlation between clinical and electrophysiological progression within any given sensory modality. The progression of clinical disability, however, showed a fairly good correlation with the overall progression of EP abnormalities. We conclude that EPs complement the neurological exam in the evaluation of MS and may have a place in the investigation of the effects of therapeutic agents on the neurological status in MS.
\end{abstract}

RÉSUMÉ: Enregistrements sériés des potentiels évoqués multimodes dans la sclérose en plaques: étude de contrôle prospective sur une période de 4 ans Dans une étude prospective d'une durée de 4 ans, on a évalué les potentiels évoqués (PEs) visuels d'inversion des patterns, auditifs du tronc cérébral et somesthésiques à latence brève du nerf médian chez vingt patients atteints de sclérose en plaques (SEP) dont le diagnostic clinique était certain. Un examen neurologique standardisé a été fait à intervalles réguliers et mis en corrélation avec les modifications des PE. La plus haute incidence des anomalies des PE se retrouve dans le système visuel suivi par les systèmes somesthésiques et auditifs. Une rechute clinique était habituellement accompagnée par une détérioration des $\mathrm{PE}$, mais une amélioration clinique survenait fréquemment sans qu'il y ait une récúpération parallèle des PE. Les changements des PE n'étaient pas toujours en relation avec les symptômes cliniques et survenaient souvent pendant des périodes de rémission en l'absence des changements cliniques. La corrélation entre la progression clinique et électrophysiologique dans chacun des modes sensitifs n'était pas significative. Cependant, il existait une assez bonne corrélation entre la progression de l'invalidité clinique et la progression globale des anomalies des PE. Nous concluons que les PE sont un complément à l'examen neurologique dans l'évaluation de la SEP et peuvent avoir leur place dans l'investigation des effets des agents thérapeutiques sur l'état neurologique dans la SEP.

Can. J. Neurol. Sci. 1986; 13:320-326

The usefulness of pattern reversal visual evoked potentials (PRVEPs), somatosensory evoked potentials (SEPs), and brainstem auditory evoked potentials (BAEPs) in the diagnosis of multiple sclerosis (MS), based on their ability to reveal clinically silent lesions, is now well established. ${ }^{.}$There have been few studies, however, to explore the value of serial evoked potential (EP) recordings in monitoring the course of MS and to relate evoked potential changes to changes in the clinical condition. Halliday et $\mathrm{al}^{2}$ found no correlation between latency prolongation of the PRVEP and visual acuity. Robinson and Rudge $^{3}$ reported that changes in BAEP latencies were more closely related to activity of the disease than to the sites of new lesions as determined clinically. Matthews and Small ${ }^{4}$ con- cluded that PRVEPs and SEPs were of value in detecting silent clinical lesions, but they could not be used to monitor the course of the disease. A similar conclusion was reached by $\mathrm{Kjaer}^{5}$ for BAEPs. Walsh et $\mathrm{al}^{6}$ found a good correlation between clinical progression and the progression of electrophysiological abnormalities, but they did not address the issue of their relative values in the follow-up of MS patients. Aminoff et $\mathrm{al}^{7}$ and Davis et $\mathrm{al}^{8}$ concluded that EPs may provide data that conflict with clinical changes and are not good indicators of disease progression. Becker and Richards, ${ }^{9}$ in a three year follow-up study of PRVEPs in 40 patients, observed a significant latency increase in 18 eyes of which 12 were clinically asymptomatic during the study interval. Four eyes showed a significant decrease

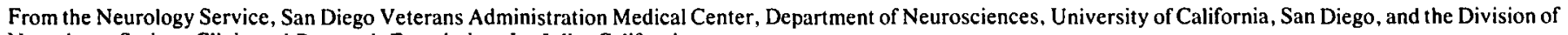
Neurology, Scripps Clinic and Research Foundation, La Jolla, California

Received March 27, 1986. Accepted June 16, 1986

Reprint requests to: V. Iragui, Department of Neurosciences (M-008), University of California, San Diego, La Jolla, CA 92093, U.S.A. 
in latency, and all of them had had an episode of optic neuritis within five weeks immediately preceding the first PRVEP test.

In this paper, we report the results of a four year prospective study designed to assess the value of SEPs, PRVEPs and BAEPs in monitoring the clinical course of MS, particularly as a method of identifying relapse and remission, and in objectively evaluating disease progression.

\section{METHODS}

We studied 20 patients with definite multiple sclerosis according to the criteria of Schumacher et al ${ }^{10}$ who participated in a clinical trial of basic myelin protein. "In the present report the results obtained from the placebo and treated groups have been pooled since there was no significant difference between the two groups. All subjects gave informed consent to participate in the study. There were 15 men and 5 women, and their ages ranged from 22 to 63 years. At entry, Kurtzke disability status score (DSS) ranged from 2 to 7 with a mean of 4.55 . The course of the disease was progressive in 12 patients, relapsing in 7 and fulminating in one. The patient with fulminating course died 14 months after entry. The last electrophysiological studies were done six weeks before death. Therefore, the completion data in this case are those obtained at that time. For the rest of the patients, follow-up time ranged from 42 to 60 months, with a mean of 50 months. Standardized neurological examinations were performed at regular intervals by the same examiner (J.R.) and converted to the numerical rating system described by Kurtzke. ${ }^{12}$ In order to compare the neurological status within functional groups with abnormalities in their corresponding EP modalities, the clinical functions of the brain stem, cerebellum, visual system and somatosensory system were rated individually on a scale from 0 to 5 following the scoring system of Kurtzke with some modifications. ${ }^{12-14}$ The visual and somatosensory groups were modified to allow rating of each eye and each arm independently, and the brain stem and cerebellar groups were combined into a single posterior fossa score. Therefore, the highest possible abnormality score that a patient could receive in visual, somatosensory or posterior fossa function was 10 .

The numerical scoring system for brain stem function was: grade $0=$ normal $;$ grade $1=$ abnormal signs without disability; grade $2=$ mild dysfunction, often in the form of nystagmus noticable to the patient; grade 3 = severe nystagmus, or weakness of extraocular muscles, or moderate dysfunction of other cranial nerves; grade $4=$ marked dysarthria, or marked signs referable to other cranial nerves such as facial diplegia, or ophthalmoplegia; grade $5=$ inability to speak or swallow.

Cerebellar function was rated as follows: grade $0=$ normal; grade 1 = abnormal signs without disability; grade $2=$ mild ataxia; grade $3=$ moderate truncal or limb ataxia; grade $4=$ severe ataxia all limbs; grade $5=$ inability to perform coordinated movements due to ataxia.

Visual function in each eye was scored as follows: grade $0=$ normal ; grade 1 = scotoma with visual acuity (corrected) better than 20/30; grade 2 = scotoma with maximal visual acuity (corrected) of 20/30 to 20/59; grade 3 = large scotoma or moderate decrease in fields, but with maximal visual acuity (corrected) of 20/60 to 20/99; grade $4=$ marked decrease of fields and maximal visual acuity (corrected) of 20/100 to 20/200; grade $5=$ maximal visual acuity (corrected) less than 20/200.
Sensory function of each upper extremity was scored as follows: grade $0=$ normal; grade $1=$ impairment of vibratory sense or graphesthesia; grade 2 = decrease in position sense and two point discrimination; grade 3 = loss of vibratory sense, severe deficit in position sense or mild impairment of touch or pain perception; grade $4=$ moderate impairment of touch or pain sensation involving the greater part of the limb; grade $5=$ loss of sensation for all modalities on the greater part of the limb.

Evoked potentials were performed every twelve months and every time there was a change of signs or symptoms. Intervals ranged from one week to twelve months. Neurological examination was performed within 24 hours of the evoked potential study. The number of battery tests performed on each patient varied from 5 to 11 (mean 7.4). The battery test included PRVEPs, median nerve SEPs, and BAEPs. SEP's could not be performed on each occasion because three patients intermittently refused the test. EPs were initially interpreted by one of us (V.I.) without knowledge of the clinical findings. Each test was scored on a 0 to 5 scale, 0 being normal and 5 being the maximal grade of abnormality. Evoked potentials to stimulation of the right and left side were rated independently, so the highest possible abnormality score for the entire EP battery was 30 ( 3 tests $\times 2$ sides $\times 5$ score). The highest possible score for a given EP modality was 10 ( 2 sides $x 5$ score).

Recording electrodes were small gold-plated disks attached with collodion. Scalp electrodes were placed in accordance with the International 10-20 system at the $\mathrm{FpZ}, \mathrm{C}_{3}{ }_{3}, \mathrm{C}^{\prime}{ }_{4}, 0_{1}, 0_{2}$, $A_{1}$ and $A_{2}$ locations. Electrodes were also placed at Erb's point and on the skin over the right patella. Impedances were maintained between 1000 and 3000 ohms.

PRVEPs were recorded to full-field monocular stimulation. A black and white checkerboard pattern was back projected on a semi-translucent screen via a revolving mirror that produced a lateral movement of one square width. The movement of the mirror took $3 \mathrm{msec}$ and occurred each 500 milliseconds. The subjects sat 1 metre in front of the screen, the stimulating field subtending $32^{\circ}$ angle, and each check subtending 38 minutes. Patients with refractive error wore corrective lenses. They were instructed to fixate at a small dot in the center of the screen. Activity was recorded from the electrodes at $0_{1}$ and $0_{2}$ with a reference electrode on FpZ (10-20 system). Two hundred reponses were averaged, and two sets of averages were obtained from each eye. Frequency response $(-3 \mathrm{db})$ was $1-100 \mathrm{~Hz}$, analysis time was $300 \mathrm{msec}$ and sampling interval $1.2 \mathrm{msec}$. PRVEPs were considered abnormal if: (1) the peak latency of the major positive deflection (PI00) exceeded the mean +3 standard deviations (SD) of normal control values (118 $\mathrm{msec}$ ), (2) the interocular P100 latency difference was higher than the mean $+3 \mathrm{SD}$ of normal controls $(8 \mathrm{msec})$; or (3) P100 was absent. Each PRVEP was scored on a scale from 0 to 5, 0 being normal and 5 being the maximal grade of abnormality. A test abnormal by more than one criteria received the highest possible score. Latency prolongations were scored according to the number of SD they exceeded the upper limit of normal: score I to 4 indicated a latency within 1 to 4 SD from the upper limit of normal respectively, 5 reflected a latency exceeding 4 SD from the upper limit of normal. Only one patient showed PRVEPs abnormalities on the basis of an excessive interocular P100 latency difference alone, and was scored as a grade 1 abnormality. Absence of $\mathrm{P} 100$ received a score of 5 . 
To obtain BAEPs, rarefaction clicks produced by applying a $0.1 \mathrm{msec}$ square wave to $8 \mathrm{ohms} \mathrm{impedance} \mathrm{shielded} \mathrm{earphones}$ were presented monaurally at a rate of 10.2 per second and an intensity of $70 \mathrm{dBSL}$. All subjects had normal or near-normal hearing. Recordings were made from vertex $(\mathrm{CZ})$ to earlobe derivations. Band pass of the recording system $(-3 \mathrm{db})$ was $100-3000 \mathrm{~Hz}$, analysis time was $10 \mathrm{msec}$ and sampling interval 48 usec. Responses to 2000 clicks were averaged at least twice for each ear. Latency and amplitude measurements were done on vertex-to-ipsilateral earlobe derivations. BAEPs were considered abnormal if: (1) the I-III, III-V, (or III-IV/V), and I-V (or I-IV/V) interpeak latencies (IPLs) exceeded the mean +3 SD of normal control values; (2) component V (or IV/V) or components III and V (or IV/V) were absent, (3) the V:I (or IV/V:I) amplitude ratio was less than 0.5 . The degree of abnormality was scored according to the number of SDs the IPLs exceeded the upper limits of normal in the same way as described for PRVEPs. When more than one IPL was prolonged, the highest score was selected. Absence of IV/V component (one case) and absence of III and IV/V waves (two cases) were scored 5. Only one test was considered abnormal solely on the basis of reduced IV/V:I amplitude ratio and received a score of 1.

SEPs were elicited by independent stimulation of each median nerve. Square wave electrical pulses of $0.1 \mathrm{msec}$ duration and sufficient intensity to produce a small visible thumb twitch were delivered unilaterally at a rate of $2.5 / \mathrm{sec}$. through a pair of disk electrodes applied longitudinally over the median nerve at the wrist with the cathode $3 \mathrm{~cm}$ proximal to the anode. Recordings were made from electrodes at the ipsilateral Erb's point and at the contralateral central region $\left(\mathrm{C}_{3}{ }_{3}\right.$ or $\left.\mathrm{C}_{4}^{\prime}\right)$, using an electrode placed on the skin over the left patella as a reference. Bandpass $-3 \mathrm{db}$ ) was $3 \mathrm{~Hz}$ to $3000 \mathrm{~Hz}$, analysis time was 30 msec and sampling interval 120 usec. Averages of 1000 responses were obtained, and each average was repeated at least twice.

SEPs were judged to be abnormal if they met one of the following criteria: (1) P9-P14 or P14-N19 interpeak latencies exceeded the mean +3 SD of control values, (2) absence of components consistently recorded in normal controls, namely P9, P14, N19 and P23, (3) Differences in interpeak latencies to left vs right median nerve stimulation which deviated from control values by at least $3 \mathrm{SD}$. Interpeak latency prolongations were scored according to the number of SD they exceeded the upper limits of normal in the same way as described for PRVEPs. Absence of wave P14 or N 19/P23 was rated 3 and absence of all waves was rated 5. P9 was present in all recordings. No SEP was abnormal solely on the basis of an increased right vs left stimulation latency difference.

An EP was reclassified to a different abnormality score on the basis of a latency change only when the change exceeded one standard deviation of the mean of normal controls. This was based on preliminary observations that small latency fluctuations that approached, but did not exceed, one standard deviation occurred from day to day in MS patients in clinical remission, particularly in those who had abnormally prolonged latencies. The data was statistically analyzed using a chi square test and a two-tailed t-test.

\section{RESULTS}

Table 1 shows the incidence of abnormal EPs. The highest incidence of abnormalities was observed in the visual modality, followed by somatosensory and finally auditory evoked potentials. EP abnormalities in all modalities were often bilateral, as can be deduced by comparing the incidence of abnormalities in the patient group with the percentage of abnormal tests that include right and left side stimulation for each modality. There was a trend for the incidence of EP abnormalities to be higher at completion than at entry. The difference, however, was not statistically significant $(p<0.05)$ for any of the EP modalities, probably because the number of patients was small.

Table 2 shows the degree of clinical and EP dysfunction at the time of entry in the study and at completion. There is a trend for the severity of all EP modalities to increase, which reached statistical significance for PRVEPs and for the global EP score. Visual, somatosensory and posterior fossa clinical scores did not change significantly, but the DSS showed significant worsening. This is accounted for by clinical deterioration in other categories, in particular in the motor system. The progression of electrophysiological abnormalities overall correlated well with the clinical progression; mean Kurtze DSS (0 to 10 scale) increased from 4.55 at entry to 6.15 at completion and mean global EP score ( 0 to 30 scale) was 12.40 at entry and 16.00 at completion.

No difference was observed in the changes within a given EP modality between patients with normal EPs at the beginning of the study and those with abnormal EPs. There was also no difference in EP changes between patients with progressive and relapsing course.

\begin{tabular}{|c|c|c|c|c|c|c|c|}
\hline \multirow[b]{3}{*}{ PRVEPs } & \multicolumn{3}{|c|}{ At Entry } & \multicolumn{4}{|c|}{ At Completion } \\
\hline & \multicolumn{2}{|c|}{$\begin{array}{c}\text { Tests } \\
(\mathrm{N}=40)\end{array}$} & $\begin{array}{l}\text { Patients } \\
(N=20)\end{array}$ & \multicolumn{2}{|c|}{$\begin{array}{c}\text { Tests } \\
(\mathbf{N}=\mathbf{4 0})\end{array}$} & \multicolumn{2}{|c|}{$\begin{array}{l}\text { Patients } \\
(\mathrm{N}=20)\end{array}$} \\
\hline & 28 & $(70 \%)$ & $17(85 \%)$ & 32 & $(80 \%)$ & 18 & $(90 \%)$ \\
\hline SEPs & 22 & $(55 \%)$ & $13(65 \%)$ & 30 & $(75 \%)$ & 16 & $(80 \%)$ \\
\hline BAEPs & 14 & (35\%) & $10 \quad(50 \%)$ & 16 & $(40 \%)$ & 11 & (55\%) \\
\hline
\end{tabular}

Table 2: Severity Score (Mean \pm SD)

\begin{tabular}{lccc}
\hline \hline & At Entry & $\begin{array}{c}\text { At } \\
\text { Completion }\end{array}$ & $\begin{array}{c}\mathbf{p} \\
\text { Value }\end{array}$ \\
\hline ELECTROPHYSIOLOGICAL & & & \\
PRVEPs & $5.00 \pm 3.50$ & $6.85 \pm 3.20$ & $<0.02$ \\
SEPs & $4.90 \pm 4.40$ & $6.25 \pm 3.85$ & $>0.05$ \\
BAEPs & $2.45 \pm 3.09$ & $2.90 \pm 3.28$ & $>0.05$ \\
ALL EPs & $12.40 \pm 6.47$ & $16.00 \pm 5.40$ & $<0.02$ \\
CLINICAL & & & \\
Visual & $1.90 \pm 2.05$ & $2.00 \pm 2.57$ & $>0.05$ \\
Somatosensory & $3.45 \pm 2.78$ & $3.65 \pm 2.63$ & $>0.05$ \\
Posterior fossa & $2.40 \pm 1.39$ & $2.95 \pm 1.98$ & $>0.05$ \\
DSS & $4.55 \pm 1.80$ & $6.15 \pm 2.35$ & $<0.002$ \\
\hline
\end{tabular}

Electrophysiological and clinical scores at entry and completion of the study. The scales range from $0-10(0=$ normal $; 10=$ maximal degree of abnormality), except for "ALL EPs", where it ranges from $0-30$. 


\section{PRVEPS}

At entry 17 patients (85\%) had abnormal PRVEPs (Table 1), 11 of whom had abnormal responses in both eyes. A total of 28 abnormal responses to monocular stimulation were obtained and there was clinical evidence of optic nerve disease by history or physical examination in 17 of these eyes $(60.7 \%)$.

At completion of the study, 18 patients $(90 \%)$ had abnormal PRVEPs, 14 of them with binocular abnormalities. Of the 32 electrophysiologically abnormal eyes, there was clinical evidence of disease in 23 of them (71.9\%), the remaining 9 eyes being entirely normal when clinical criteria alone were considered. Of the 8 eyes with normal reponses, there was evidence of clinical involvement in 2 of them.

Clinical evidence of optic neuritis existed in 25 eyes (62.5\%) at completion of the study. Clinical criteria for optic neuritis included either a definite history of transient visual loss or the presence of optic atrophy, central scotoma, or red color desaturation in ophthalmological examination. Visual acuity was normal in 9 eyes $(36 \%), 20 / 25$ to $20 / 30$ in $7(28 \%), 20 / 40$ to $20 / 100$ in $7(28 \%)$, and worse than $20 / 100$ in $2(8 \%)$. PRVEPs were abnormal in $23(92 \%)$ of the 25 eyes with clinical evidence of optic neuritis, but normal in the remaining $2(8 \%)$. The visual acuity in these two eyes was, respectively, 20/20 and 20/30.

Clinical relapse followed by recovery occurred in 4 eyes. A parallel deterioration in PRVEPs occurred in all cases, and consisted of a prolongation in latency noted in all eyes and a concurrent decrease in amplitude observed in 2 eyes. The PRVEP latency changes in 3 eyes persisted in spite of complete recovery of visual acuity but amplitude returned to baseline. In one eye, PRVEP latency returned to baseline although the electrophysiological recovery lagged the clinical improvement by approximately two months.

Conversely, transient PRVEP changes without concomitant visual acuity deterioration occurred in 11 eyes. These changes did not bear any detectable relationship to clinical relapse or remission. These changes were not random or erratic but showed deterioration and subsequent gradual recovery in a stepwise manner that could be followed in serial studies.

Table 3 compares the clinical changes in visual function with PRVEP changes over four years. There was no significant correlation between clinical and electrophysiological changes $(p<0.05)$. When the visual function deteriorated, PRVEPs either remained the same or worsened. Clinical improvement in visual function was followed by either an improvement or no change in PRVEPs, with one exception in which the visual evoked potential deteriorated. More important, 8 (34.8\%) of 23

\begin{tabular}{|c|c|c|c|c|}
\hline \multirow[t]{2}{*}{ CLINICAL } & \multicolumn{3}{|c|}{ PRVEPS } & \multirow[b]{2}{*}{ TOTAL } \\
\hline & Improved & Same & Deteriorated & \\
\hline Improved & 3 & 6 & 1 & 10 \\
\hline Same & 5 & 10 & 8 & 23 \\
\hline Deteriorated & 0 & 4 & 3 & 7 \\
\hline TOTAL & 8 & 20 & 12 & 40 \\
\hline
\end{tabular}

Evolution of clinical and electrophysiological visual scores from beginning to end of the follow-up period. The figures represent the number of eyes. There was no significant correlation $(p>0.05)$ between clinical and electrophysiological changes. eyes in which the clinical visual function did not change, showed deterioration in the PRVEPs. Conversely, 8 (75\%) of 12 eyes in which PRVEPs deteriorated showed no clinical change, and only 10 (50\%) of 20 eyes in which PRVEPs did not change remained clinically the same. Of the 8 eyes that showed PRVEP improvement, 4 had had clinical optic neuritis with onset within two months prior to the first PRVEP test, and 3 of these also showed clinical improvement. These 4 eyes showed PRVEP improvement within the first six months of the study. Two of the remaining 4 eyes had evidence of optic neuritis on neuroophthalmological examination in spite of the absence of clinical history, and 2 had a normal exam and no history of optic neuritis.

\section{SEPS}

At the beginning of the study 13 patients $(65 \%)$ had SEP abnormalities (Table 1) which were bilateral in 9 of them. At completion of the study 16 patients $(80 \%)$ had abnormal SEPs, 14 of whom had abnormal responses on both sides. Among these patients the sensory examination of the extremities with SEP abnormalities was entirely normal in 6 extremities (20\%), whereas 8 extremities $(26.6 \%)$ had only mild impairment of vibratory sense, $4(13.3 \%)$ had mild touch and pain sensation impairment, 8 (26.6\%) had impairment of both touch/pain and vibratory senses and only 4 arms (13.3\%) had abnormalities in position sense, graphesthesia, and/or two-point discrimination, which were always accompanied by decrease in vibratory sensation, and in 2 of the arms also by a mild impairment of touch and pain sensations. Of the $10 \mathrm{arms}$ with normal SEPs, there was mild to moderate decrease in pain/temperature sensations in 3 of them and vibratory sense was decreased in 2 , the remaining 5 arms showing no evidence of sensory impairment. Position sense, graphesthesia and two-point discrimination were intact in every extremity that had normal SEPs.

Transient sensory deficit followed by recovery was observed in 7 arms. In 4 extremities, the sensory deficit involved paid and/or touch modalities, and was accompanied by numbness and paraesthesias. SEPs did not change in 3 of these arms, while one arm exhibited a transient SEP abnormality that recovered completely and consisted of a marked attenuation in amplitude of the P15 potential. In the remaining 3 arms the clinical relapse consisted of transient deficit in position sense, which was accompanied by decrease in graphesthesia and two-point discrimination in 2 of them. In each of these 3 arms SEPs exhibited deterioration that parallelled the clinical worsening but that was not followed by recovery when the symptoms subsided. In one of these cases the abnormality consisted of absence of the N19/P23 potentials, in the remaining two both the N19/P23 and the P15 components became undetectable.

Conversely, transient SEP changes without concomitant sensory symptoms or signs occurred in 8 arms. These changes were not random or erratic but showed stepwise deterioration and subsequent gradual recovery that could be followed in serial studies.

Comparison of the somatosensory system score at entry and completion of the study (Table 4) showed a significant increase in sensory deficit in 17 arms, clinical improvement in 12 arms, and no change in the remaining 11 arms. Of the 17 arms with increased sensory deficit, SEPs had deteriorated in 7, remained unchanged in 9 and improved in one. Eight of the 12 arms with 


\begin{tabular}{lcccc}
\hline Table 4: Somatosensory Function & & \\
\hline \hline CLINICAL & \multicolumn{4}{c}{ SEPs } \\
\cline { 2 - 5 } & Improved & Same & Deteriorated & TOTAL \\
\hline Improved & 2 & 8 & 2 & 12 \\
Same & 0 & 5 & 6 & 11 \\
Deteriorated & 1 & 9 & 7 & 17 \\
TOTAL & 3 & 22 & 15 & 40 \\
\hline
\end{tabular}

Evolution of clinical and electrophysiological somatosensory scores from beginning to end of the follow-up period. The figures represent number of arms. There was no significant correlation ( $p>0.05$ ) between clinical and electrophysiological changes.

improved sensory score showed no SEP change. Of the 11 arms that remained clinically unchanged, the SEP was worse in 6 of them. Compared to entry score, the completion SEP score had worsened in 15 arms, remained the same in 22 and improved in 3. Of the 15 arms with worsened SEPs, only 7 (47\%) showed clinical deterioration, while $6(40 \%)$ remained clinically unchanged and $2(13 \%)$ improved. Only $5(23 \%)$ of 22 arms in which SEPs did not change remained clinically unchanged, while $8(36 \%)$ improved and $9(41 \%)$ deteriorated. Two of the 3 arms with improved SEPs showed clinical improvement in position sense, and one worsened due to impairment in pain sensation. There was no significant correlation between clinical and electrophysiological changes $(P<0.05)$.

\section{BAEPs}

At entry 10 patients (50\%) had abnormal BAEPs (Table 1) which were binaurally abnormal in 4 of them. At completion BAEPs were abnormal in 11 patients $(55 \%), 5$ of them with binaural abnormalities. Clinical evidence of brainstem and/or cerebellar dysfunction was present in 8 of the 11 patients with abnormal BAEPs and in 6 of the 9 patients with normal BAEPs.

Transient BAEP changes without clinical brainstem and/or cerebellar changes occurred in only 2 patients. Clinical relapses involving brainstem and/or cerebellar function followed by complete or partial recovery occurred in nine instances. BAEPs did not change in 5, exhibited a parallel deterioration in 3 and a paradoxical improvement on one occasion. Of the three instances in which BAEPs deteriorated there was subsequent improvement in two cases. Of particular interest is a patient with an episode of right trigeminal neuralgia of two weeks duration that was accompanied by BAEP abnormalities from the ear ipsilateral to the pain. Prior to the onset of pain, BAEPs showed a mild I-V interpeak latency prolongation $(4.9 \mathrm{msec})$. On the second day of trigeminal neuralgia only wave I could be recorded. After two weeks, as the pain subsided, wave I remained unchanged and wave $\mathrm{V}$ was again obtained with a very prolonged latency $(\mathrm{I}-\mathrm{V}$ interpeak latency $=7.1 \mathrm{msec})$. Three months later, the latency of wave $\mathrm{V}$ and the I-V interpeak latency $(5.0$ msec) had almost returned to normal. This patient has been reported in detail. ${ }^{15}$

Comparison of the combined brainstem and cerebellar score at entry and completion of the study (Table 5) showed an increased deficit in 8 patients, no change in 9 , and improvement in 3 . Of the 8 patients with clinical posterior fossa function deterioration, BAEPs did not change in 4, deteriorated monau-

\begin{tabular}{|c|c|c|c|c|c|}
\hline \multirow[t]{2}{*}{ CLINICAL } & \multicolumn{3}{|c|}{ BAEPs } & \multirow[b]{2}{*}{ TOTAL } & \\
\hline & Improved & Same & Deteriorated & & \\
\hline Improved & 1 & 4 & 1 & 6 & (3 patients) \\
\hline Same & 2 & 12 & 4 & 18 & (9 patients) \\
\hline Deteriorated & 2 & 12 & 2 & 16 & (8 patients) \\
\hline TOTAL & 5 & 28 & 7 & 40 & (20 patients) \\
\hline
\end{tabular}

Evolution of posterior fossa (combined brainstem and cerebellar) clinical scores and BAEP scores from beginning to end of the follow-up period. BAEP figures represent the number of ears. Each clinical score is represented twice, since it was matched with BAEPs from each year. There was no significant correlation $(P>0.05)$ between clinical and electrophysiological changes.

rally in 2 , and improved monaurally in 2 . Of the 3 patients in whom clinical brainstem function improved, BAEPs did not change in one, deteriorated monaurally in one and improved monaurally in one. Clinical brainstem function remained unchanged in 9 patients, and BAEPs did not change in 4 , deteriorated monaurally in 2 and binaurally in one, and improved monaurally in the remaining 2. Comparison of BAEP score at entry and completion showed improvement in 5 patients, no change in 9 and deterioration in 6. The BAEP improvement occurred monaurally in all 5 patients, of whom 2 showed no clinical change in posterior fossa function, 2 deteriorated and one improved. BAEP deterioration occurred binaurally in one patient who did not experience clinical posterior fossa changes and monaurally in 5, of whom one was clinically improved, 2 remained unchanged, and 2 deteriorated. BAEPs remained binaurally unchanged in 9 patients, of whom one was clinically improved, 4 were the same and 4 deteriorated. No patient showed improvement of BAEPs from one ear and deterioration of BAEPs from the opposite ear. There was no significant correlation between clinical and electrophysiological changes $(p<0.05)$.

\section{Discussion}

The incidence of EP abnormalities in the present study, highest within the visual system followed by the somatosensory and auditory systems respectively, is in agreement with many previous reports.' The incidence of SEP abnormalities tends to approach that of PRVEP abnormalities when both upper and lower extremity SEPs are tested, rather than using only upper extremities SEPs as was the case in the present study. ${ }^{1}$ This study also confirms the well-known fact that EPs can reveal subclinical lesions and that combined evoked potential studies increase the yield of EP abnormalities. In addition, the present investigation demonstrates that evoked potentials can detect subclinical progression and relapses of the disease. Evoked potentials are helpful in documenting involvement of a sensory system when symptoms are lacking or trivial, and in quantifying the degree of abnormality.

Clinical exacerbations were often accompanied by electrophysiological changes in the corresponding sensory modality. All four instances of acute relapses involving the visual system were accompanied by PRVEP deterioration. Within the somatosensory system, the electroclinical correlation was limited to 
involvement of position sense, graphesthesia and/or two-point discrimination. Disturbances of touch or pain sensation were usually not accompanied by SEP changes. This is in agreement with the widely accepted view that the components of the SEP measured in clinical testing are primarily mediated by the posterior column-lemniscal system. 'BAEP changes showed a relationship to clinical exacerbations in only one-third of relapses involving the brainstem or cerebellum, which is not unexpected since cerebellar and brainstem involvement may occur with sparing of the auditory pathways. Hearing impairment did not occur in any of the patients which is in keeping with observations that central BAEP abnormalities are usually associated with normal hearing as tested by conventional audiometry.' Psychophysical data, nevertheless, suggest that subtle auditory problems that may not always be detectable by conventional clinical testing frequently occur in MS. ${ }^{14.15}$ Such subtle auditory deficits may have been overlooked in our patients.

Electrophysiological deterioration has been reported to occur during periods of exacerbation regardless of whether the relapse clinically involved the corresponding EP pathways. ${ }^{3,4,18.19}$ We found no relationship between changes in a given EP modality and clinical exacerbations involving other sites.

Unlike clinical exacerbations, remission of symptoms was often unaccompanied by electrophysiological improvement and EP abnormalities frequently persisted after clinical recovery had taken place. In addition, electrophysiological deterioration, both transient and long-lasting, often occurred in the absence of any detectable clinical symptoms or signs. These changes can not be attributed to the excessive intertrial variability that often occurs in MS patients and was observed in some of our tests. $3,7,20$ They were not random or erratic changes but occurred in a stepwise manner that could be followed in serial studies. Electrophysiological improvement also took place in the absence of clinical change, in particular in the auditory and visual modalities. Four of 5 patients with monaural BAEP improvement showed either no change or deterioration of clinical posterior fossa function. Of 5 eyes showing PRVEP improvement without clinical change, one had suffered the onset of acute clinical optic neuritis two months prior to entry into the study, 2 had evidence of optic neurities on neuroophthalmological examination but no history of optic neuritis, and 2 were neuroophthalmologically normal. This is in contrast with the $4 \mathrm{MS}$ patient eyes that showed PRVEP latency reductions in the study of Becker and Richards, ${ }^{9}$ all of whom had had the onset of acute clinical optic neuritis within five weeks immediately preceding the first PRVEP test.

Thus, there was a lack of clear correlation between clinical and electrophysiological abnormalities. Evoked potential changes occurred in the absence of clinical changes and, conversely, clinical deterioration or improvement took place without parallel EP changes (Tables 3-6). In general, however, the progression of electrophysiological abnormalities correlated with the clinical progression of the disease. The overall clinical disability (DSS) increased from a mean of 4.55 to 6.15 over a four year period, and the overall electrophysiological function deteriorated from 12.40 to 16.00 (Table 2). Similar findings have been reported by Walsh et al. ${ }^{6}$

Some latency fluctuation occurs from one day to the next in the EPs of normal controls and MS patients in remission, ${ }^{1.9}$ in particular in the visual modality. Small changes in stimulus parameters, fluctuations in body temperature and other physio- logical and technical factors may contribute to these changes. The EP scoring system used in this study attaches significance only to latency changes that exceed one standard deviation from the mean value of normal controls which, in our experience, is beyond the day to day fluctuations observed in MS patients in remission. We concur with Becker and Richards ${ }^{9}$ that attaching significance to small latency changes is highly questionable.

The lack of a close relationship between clinical and electrophysiological abnormalities cannot be construed as evidence against the usefulness of EPs in the evaluation of disease progression in MS, as has been suggested by some authors. ${ }^{4.7}$ Clinical changes are not good indicators of the progression of this disease and discrepancies often occur between clinical and pathological findings. In fact, the lesions seen at autopsy almost invariably greatly outnumber those suspected on the basis of clinical symptoms and signs. Furthermore, lesions have been demonstrated at autopsy in asymptomatic patients in areas from which one would expect abnormal signs and symptoms to arise. ${ }^{21}$ Whether subclinical EP changes reflect additional pathologic lesions or changes in conduction in previously diseased fiber tracts is not known. Although electropathological correlations cannot be established in our patients, the present study indicates that EPs provide information complementary to the clinical exam, and it seems reasonable to postulate that clinical and electrophysiological changes together may reflect disease activity with greater accuracy than clinical changes alone. The information complementary to the clinical exam provided by EPs may be particularly relevant in the serial evaluation of MS patients undergoing therapeutic trials, in whom close follow-up of disease progression is essential. ${ }^{22-29}$

\section{ACKNOWLEDGEMENTS}

We would like to express our appreciation to Ms. Barbara Reader for her assistance in the preparation of this manuscript. This was supported in part by the National Multiple Sclerosis Society and Research Service, San Diego VAMC.

\section{REFERENCES}

1. Chiappa KH. Evoked potentials in clinical medicine. Raven Press, New York, 1983.

2. Halliday A, McDonald W, Mushin J. Visual evoked response in diagnosis of multiple sclerosis. Br Med J 1973; 4: 661-664.

3. Robinson K, Rudge P. The stability of the auditory evoked potentials in normal men and in patients with multiple sclerosis. J Neurol Sci 1978; 36: 147-156.

4. Matthews WB, Small DG. Serial recording of visual and somatosensory evoked potentials in multiple sclerosis. J Neurol Sci 1979; 40: 11-21.

5. Kjaer $M$. Variations of brain stem auditory evoked potentials correlated to duration and severity of multiple sclerosis. Acta Neurol Scand 1980; 61: 157-166.

6. Walsh JC, Garrick R, Cameron J, et al. Evoked potential changes in clinically definite multiple sclerosis: a two year follow up study. J Neurol Neurosurg Psychiat 1982; 45: 494-500.

7. Aminoff MJ, Davis SL, Panitch HS. Serial evoked potential studies in patients with multiple sclerosis. Clinical relevance. Arch Neurol 1984; 41: 1197-1202.

8. Davis SL, Aminoff MJ, Panitch HS. Clinical correlations of serial somatosensory evoked potentials in multiple sclerosis. Neurology 1985; 35: 359-365.

9. Becker WJ, Richards MI. Serial pattern shift visual evoked potentials in multiple sclerosis. Can J Neurol Sci 1984; 11 : 53-59.

10. Schumacher GA, Beebe G, Kibler RF, et al. Problems of experimental trials of therapy in multiple sclerosis: report by a panel on evaluation of experimental trials of therapy in multiple sclerosis. Ann NY Acad Sci 1965; 122: 552-568. 
11. Romine JS, Salk J. A study of myelin basic protein as a therapeutic probe in patients with multiple sclerosis. In: Hallpike JF, Adams CWM, Tourtellote WW, eds. Multiple Sclerosis. Pathology, Diagnosis and Management. Baltimore: Williams and Wilkins, 1983: 621-630.

12. Kurtzke FK. Further notes on disability evaluation in multiple sclerosis with scale modifications. Neurology 1965; 15: 654-661.

13. Kurtzke JF. Neurologic impairment in multiple sclerosis and the disability status scale. Acta Neurol Scand 1970; 46: 493-512.

14. Rose AS, Kuzma JW, Kurtzke JF, et al. Cooperative study in the evaluation of therapy in multiple sclerosis: ACTH vs placebo in acute exacerbations. Preliminary Report. Neurology 1968; 18 (No. 6, Part 2): 1-19.

15. Iragui VJ, Wiederholt WC, Romine JS. Evoked potentials in trigeminal neuralgia associated with multiple sclerosis. Arch Neurol 1986; 43: 444-446.

16. Reagan D. Psychophysical tests of vision and hearing in patients with multiple sclerosis. Adv Neurol 1981; 31: 217-257.

17. Mustillo P. Auditory deficit in multiple sclerosis: a review. Audiology 1984; 23: 145-164.

18. Confavreux C, Mauguiere F, Courjon J, et al. Course of visual evoked potentials in multiple sclerosis: Electroclinical correlations and pathophysiological considerations in 25 patients. In: Courjon J, Mauguiere F, Revol M, eds. Clinical Applications of Evoked Potentials in Neurology. New York: Raven Press, 1982; $541-550$.

19. Likosky W, Elmore RS. Exacerbation detection in multiple sclerosis by clinical and evoked potential techniques: A preliminary report. In: Courjon J, Mauguiere F, Revol M, eds. Clinical Applications of Evoked Potentials in Neurology. New York: Raven Press, 1982: 535-540.
20. Cohen SN, Syndulko K, Hansch E, et al. Variability on serial testing of visual evoked potentials in patients with multiple sclerosis. In: Courjon J, Mauguiere F, Revol M, eds. Clinical Applications of Evoked Potentials in Neurology. New York: Raven Press, 1982; 559-565.

21. Poser CM. Exacerbations, activity and progression in multiple sclerosis, Arch Neurol 1980; 37: 471-474.

22. Dau PC, Petajan JH, Johnson DP, et al. Plasmapheresis in multiple sclerosis: preliminary findings. Neurology 1980; 30: 1023-1028.

23. Rosen AD, Hamburger MI. Plasmapheresis in multiple sclerosis: effect on the visual evoked potential. Plasma Ther 1981;2: 239-242.

24. McFarlin DE, McFarland HF. Multiple sclerosis. N Engl J Med 1982; 307: 1183-1188, 1246-1251.

25. Mertin J, Rudge $P$, Kremer M, et al. Double-blind, controlled trial of immunosuppression in the treatment of multiple sclerosis: final report. Lancet 1982; 2: 351-354.

26. Dau PC. Plasmapheresis. Therapeutic or experimental procedure? Arch Neurol 1984; 41: 647-653.

27. Noseworthy JH, Seland TP, Ebers GC. Therapeutic trials in multiple sclerosis. Can J Neurol Sci 1984; 11: 355-362.

28. Knobler RL, Panitch HS, Braheny SL, et al. Systemic alphainterferon therapy of multiple sclerosis. Neurology 1984; 34: 1273-1279.

29. Gordon PA, Carroll DJ, Etches WS, et al. A double-blind controlled pilot study of plasma exchange versus sham apheresis in chronic progressive multiple sclerosis. Can J Neurol Sci 1985; 12: $39-44$. 